\title{
A case of adaptation through a mutation in a tandem duplication during experimental evolution in Escherichia coli
}

Ram P Maharjan ${ }^{1+}$, Joël Gaffé ${ }^{2,3+}{ }^{+}$, Jessica Plucain ${ }^{2,3}$, Martin Schliep ${ }^{1}$, Lei Wang ${ }^{4,5}$, Lu Feng ${ }^{4,5}$, Olivier Tenaillon ${ }^{6,7}$, Thomas Ferenci $^{1+}$ and Dominique Schneider ${ }^{2,3^{*}+}$

\begin{abstract}
Background: DNA duplications constitute important precursors for genome variation. Here we analyzed an unequal duplication harboring a beneficial mutation that may provide alternative evolutionary outcomes.

Results: We characterized this evolutionary event during experimental evolution for only 100 generations of an Escherichia coli strain under glucose limitation within chemostats. By combining Insertion Sequence based Restriction Length Polymorphism experiments, pulsed field gel electrophoresis and two independent genome re-sequencing experiments, we identified an evolved lineage carrying a $180 \mathrm{~kb}$ duplication of the $46^{\prime}$ region of the E. coli chromosome. This evolved duplication revealed a heterozygous state, with one copy harboring a 2668 bp deletion that included part of the ogrK gene and both the yegR and yegS genes. By genetically manipulating ancestral and evolved strains, we showed that the single yegS inactivation was sufficient to confer a frequency dependent fitness increase under the chemostat selective conditions in both the ancestor and evolved genetic contexts, implying that the duplication itself was not a direct fitness contributor. Nonetheless, the heterozygous duplicated state was relatively stable in the conditions prevailing during evolution in chemostats, in striking contrast to non selective conditions in which the duplication resolved at high frequency into either its ancestral or deleted copy.

Conclusions: Our results suggest that the duplication state may constitute a second order selection process providing higher evolutionary potential. Moreover, its heterozygous nature may provide differential evolutionary opportunities in alternating environments. Our results also highlighted how careful analyses of whole genome data are needed to identify such complex rearrangements.
\end{abstract}

Keywords: Experimental evolution, Chemostats, Escherichia coli, Duplication, Heterozygous duplication, Insertion sequence elements, Frequency-dependent selection, Indirect selection

\section{Background}

Chromosomal duplications influence adaptive processes by potentially impacting on either short-term or longterm evolutionary outcomes. In the short term, duplications may confer higher fitness owing to increased gene dosage [1-3]. Indeed, they overcome limited expression of specific functions when microbial populations evolve for instance under conditions of lactose limitation in

\footnotetext{
* Correspondence: dominique.schneider@ujf-grenoble.fr

${ }^{\dagger}$ Equal contributors

${ }^{2}$ Laboratoire Adaptation et Pathogénie des Micro-organismes, Université Joseph Fourier Grenoble, BP 170, F-38042, Grenoble cedex 9, France

${ }^{3}$ CNRS UMR5163, F-38042, Grenoble cedex 9, France

Full list of author information is available at the end of the article
}

Escherichia coli $[1,4]$, glucose limitation in yeast [5,6], or antibiotic pressure in Salmonella [2,3]. Moreover, the flexibility associated with the potential transient nature of duplication events may provide evolutionary advantages in fluctuating environments or relaxed selection [1]. Over longer evolution, duplication events can enhance survival abilities by buffering against deleterious mutations [7]. They may also provide important pathways to genetic innovation, opening opportunities for the emergence of novel functions and the expansion of gene families $[6,8,9]$. Genome sequences have confirmed the contribution of DNA duplications to evolutionary processes $[8,10]$. Gene duplication may be related to

\section{Biomed Central}


evolutionary divergence [11] by providing intermediates during the duplication-divergence process [12]. Different models have been proposed to identify the selective forces for gene duplication, including a neutral event followed by accumulation of mutations [8], the evolution of intermediates with secondary functions [13], and complementary loss-of-functions in each of the duplicated copies [14]. Chromosomal duplication is associated with the potential for increased genetic variation that may result in an equilibrium between organismal robustness against deleterious mutations and evolvability with potentially beneficial mutations that may arise in a copy of a duplicated region, thereby contributing to evolved phenotypes.

Most information on evolution through duplication relies on retrospective comparative analyses of available genomes [8]. However, direct observation of the rise and segregation of duplications is much scarcer owing to their rarity, instability and the subtle phenotypic effects they may confer. Bacterial evolution experiments [15] provide opportunities to identify intermediates during duplication processes. However, only few duplication events have been detected in these experiments, except during high temperature adaptation [16] where evolved clones contained on average 0.2 large duplication compared to 9.2 SNPs and short indels [17]. Most mutations identified during laboratory evolution experiments include SNPs and short indels [18-21] that have been shown to explain many evolved phenotypes. Consequently, little information is available over short time-frames on the dynamics of duplication processes, including the divergence of duplicated copies. Hence, to the best of our knowledge, no data with co-linear wild-type and mutant alleles are available during evolution experiments, whereas duplications have been commonly found by classical genetics in Salmonella populations adapting to diverse conditions including nutrient-limited chemostats [3,22].

We designed evolution experiments during which an $E$. coli ancestral strain was propagated as large populations ( $>10^{10}$ bacteria) for $\sim 100$ generations within chemostats under glucose or phosphate limitation [23,24]. Chemostats are continuous culture systems in which the bacteria deplete substrates but are maintained in constant population numbers over extended periods. Mutations improve fitness in this environment, allowing population sweeps. The initial selection is for better utilization (transport and scavenging) of limiting nutrient but, after 50 generations, populations diversify into separate lineages with diverse phenotypes and eventually evolve cross-feeding polymorphisms [19,23-26]. Many SNPs, deletions, IS movements and short indels have been identified that explained the functional changes that occurred in different phenotypic groups $[19,27]$. However, the genetic bases of the metabo- lic changes and fitness benefits of some groups of clones have not yet been identified. In particular, one group of clones producing unusually elevated acetate levels and reduced growth yield is not characterized by the typical improved glucose transport [28] and lacks all the regulatory changes identified so far in other clones from the same populations [23].

Here, we combined Insertion Sequence-based Restriction Length Polymorphism, pulsed-field gel electrophoresis and genome re-sequencing to characterize one representative of this group of clones. We identified a novel chromosomal duplication, one copy of which was altered by a deletion that conferred higher fitness under the conditions prevailing in the chemostat. This duplication may constitute an evolutionary intermediate [12]. Hence, the duplication is readily resolved into a single copy, either ancestral or evolved, the resolution frequency being higher under non-selective conditions. Owing to its instability, we had difficulties to detect the duplication by genome re-sequencing. Hence, we missed the duplication during a first genome re-sequencing attempt of the focal clone. Only the discrepancy between the data generated by our combined approaches led us to carefully re-examine the genome and to identify the duplication. This instability may explain the underrepresentation of large duplications observed during laboratory bacterial evolution experiments.

\section{Results}

\section{Initial characterization of BW4005}

The evolved clone BW4005 was previously characterized phenotypically by comparison to its ancestor, and exhibited a lower growth yield, a higher production of acetate from glucose in chemostat culture, and a lack of obvious difference in glucose uptake [28]. At the ecological level, BW4005 was present in low proportion in its glucose-limited evolving population and displayed negative frequency-dependent fitness when competed with the ancestor [19]. By contrast, no genetic information was available to explain these phenotypic and ecological traits, even after re-sequencing the genome of a clone, called BW4005.1, recovered from the initial glycerol stock of BW4005, by using a combination of 454 and Illumina technologies. Indeed, we detected a single mutation compared to the ancestor, consisting of a short deletion in $a h p C$ encoding the alkyl hydroperoxide subunit involved in peroxide resistance (Table 1). As shown below, this mutation did not contribute to increased fitness in the chemostats. Moreover, the genome sequence of BW4005.1 was inconsistent with previously detected changes in the RFLP profiles of another clone isolated independently from the same glycerol stock of BW4005, called BW4005.2, using the mobile genetic element IS3 as a probe [27]. Indeed, while the RFLP-IS3 profile of the 
Table 1 List of mutations found in BW4005.1 and BW4005.C6

\begin{tabular}{lllll}
\hline Event number & Genome position ${ }^{\mathbf{a}}$ & Gene name & Type & Present in $^{\mathbf{b}}$ \\
\hline 1 & 541,030 & ahpC & 3-bp deletion & BW4005.1 \\
& & & BW4005.C6 \\
\hline 2 & $2,058,010-2,060,677$ & ogrK-yegS-yegR & 2668-bp deletion & BW4005.C6 \\
\hline 3 & $1,993,429-2,172,740$ & NA & $\sim 180$-kb duplication & BW4005.C6 \\
\hline
\end{tabular}

${ }^{\mathrm{a}}$ The positions are given according to the genome sequence of the ancestor strain BW2952 [29]. ${ }^{\mathrm{b}}$ The genome sequence of BW4005.1 was first obtained and detected only the first mutation, while the one of BW4005.C6 was obtained afterwards and contained the three mutations. ${ }^{\mathrm{C}}$ Not applicable.

ancestral strain BW2952 revealed four IS3-containing EcoRV genomic fragments of $1.7,2.6,4.6$, and $10.6 \mathrm{~kb}$, respectively, with hybridization intensities proportional to the relative molecular weight of these fragments (Figure 1a), the one of BW4005.2 showed a fifth hybridizing fragment of $\sim 7.9 \mathrm{~kb}$ with a hybridization intensity stronger than expected from its molecular weight (Figure 1a). This was unlikely to be due to contamination, since both clones harbored the $a h p C$ mutation (Table 1).

\section{Genomic rearrangements in BW4005}

We characterized the genomic sequences adjacent to IS3 in the $7.9-\mathrm{kb}$ fragment specific to BW4005.2 by inverse PCR experiments. One side (right on Figure 1b) corresponded to the gatD gene with the IS3 insertion at exactly the same position $(2,061,936)$ as observed in the ancestor for the IS3 element contained in the 10.6-kb hybridizing fragment. This particular ancestral IS3 is inserted between positions 2,060,677 and 2,061,936. The other side corresponded to a genomic sequence $(2,058,010)$ located 2668 bp upstream in the ancestral genome. Therefore, this fifth IS3 probably resulted from an IS3 transposition event at position 2,058,010 followed by a recombination event between this new IS3 copy and the one located in the ancestral genome between positions 2,060,677 and 2,061,936 within gatD. These events subsequently resulted in the 2668-bp deletion of the intervening fragment, affecting the two genes yegR and yegS and part of $\operatorname{ogrK}$ (Figure 1c). However, two features of the RFLP-IS3 pattern suggested a more complex rearrangement (Figure 1a): the two 10.6 and $7.9 \mathrm{~kb}$ IS3-hybridizing fragments were co-existing in BW4005.2, indicating the presence of two copies of at least gatD, and the $7.9 \mathrm{~kb}$ fragment presented a hybridizing intensity higher than expected from its molecular weight compared to the four other hybridizing fragments. Altogether, these data might be explained by the presence in the chromosome of BW4005.2 of a tandem duplication containing two heterozygous copies, with and without the IS3-generated deletion respectively (Figure 1c). We then performed PFGE experiments with XbaI-digested genomic DNA of BW2952 and BW4005.2 (Figure 1d). We found two extra XbaI fragments of 61.5 and $19 \mathrm{~kb}$ in BW4005.2 compared to BW2952, which also suggested the presence of a complex rearrangement.
These genomic rearrangements were not detected by genome sequencing in the clone BW4005.1. Since both clones BW4005.1 and BW4005.2 were derived from a single cycle of growth after plating the initial glycerol stock of BW4005 onto LB solid medium, we hypothesized that the rearrangements were present in the original evolved clone, but were characterized by a high instability. We therefore investigated the stability of the genomic rearrangements by isolating six additional clones, called BW4005.C1 to BW4005.C6, after plating the initial glycerol stock of BW4005 onto LB medium, and analyzing them by RFLP-IS3 and PFGE (Figure 1e). The RFLP-IS3 profiles were checked for the presence or absence of the 10.6 and $7.9 \mathrm{~kb}$ IS3-hybridizing fragments. Hence, their simultaneous presence was diagnostic of the putative heterozygous duplication while the presence of only the first or second fragment indicated the resolution of the putative duplication into the ancestral or deleted copy, respectively. Similarly, the PFGE profiles were checked for the presence or absence of the two 61.5 and $19 \mathrm{~kb}$ bands. The RFLP-IS3 profiles revealed that four (BW4005.C1 to BW4005.C4) of the six clones had lost either one of the putative duplicated DNA copies (Figure 1e), with clones BW4005.C1 and BW4005.C2 harboring the single deleted copy and BW4005.C3 and BW4005.C4 the ancestral copy. The two last clones, BW4005.C5 and BW4005.C6, retained the putative heterozygous duplication. Consistent with the RFLP-IS3 profiles, the PFGE analysis showed that clones BW4005.C1 and BW4005.C2 retained only the 19 $\mathrm{kb}$ band, while BW4005.C3 and BW4005.C4 had the ancestral profile, and BW4005.C5 and BW4005.C6 harbored the two extra fragments (Figure 1e). These results indicated the high genetic instability of the putative duplication as well as its capacity for resolution into alternative states.

Moreover, both RFLP and PFGE experiments, using genomic DNA isolated from different independent cultures of BW4005.C6, exhibited variation in the intensity of the additional bands (Figure 1 and data not shown), which further suggested the instability of the genetic rearrangement. This instability may provide an explanation for the discrepancies between the RFLP/PFGE experiments and the genome re-sequencing results and is considered in more detail below. 

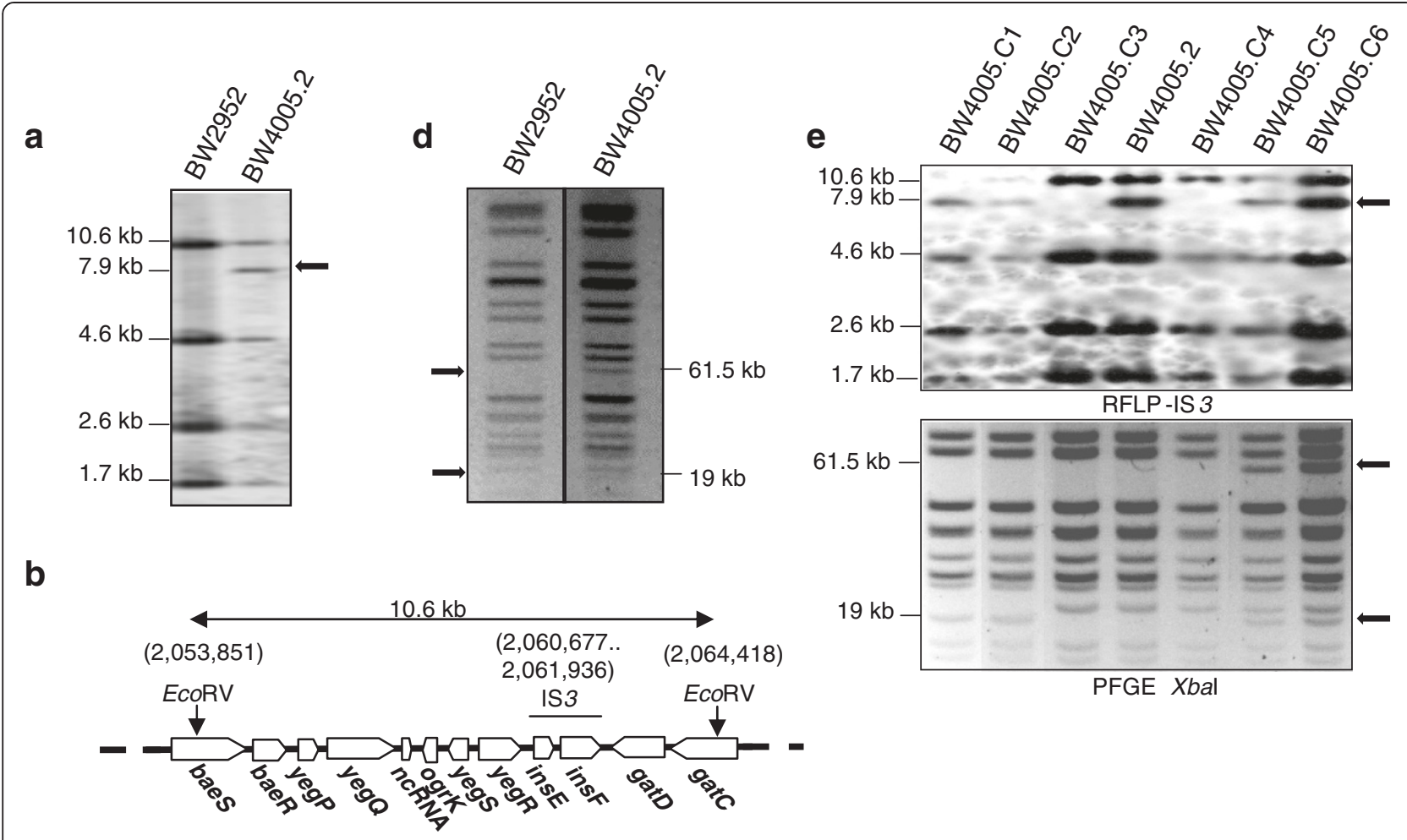

C
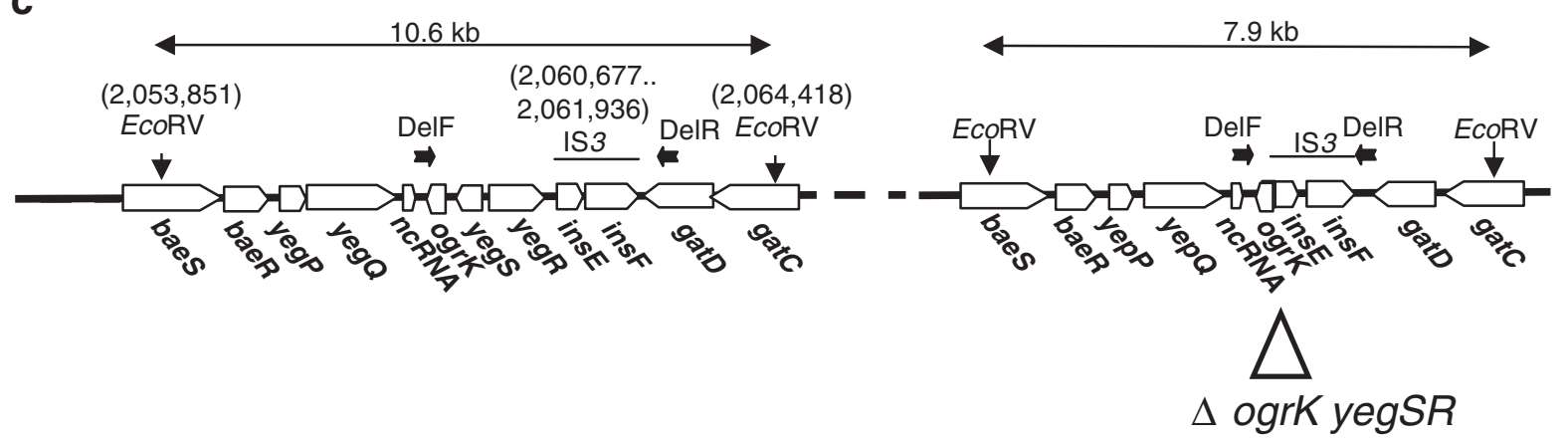

Figure 1 Genomic rearrangements in the evolved clone BW4005 sampled from a glucose-limited chemostat. (a) RFLP-IS3 of EcoRVdigested genomic DNA of BW2952 and BW4005.2 [24]. A fifth hybridizing fragment (arrow) with stronger hybridization intensity was detected in BW4005.2. (b,c) Maps showing the genomic regions corresponding to the 10.6 and $7.9 \mathrm{~kb}$ EcoRV IS3-hybridizing fragments, including the sequences adjacent to IS3 in BW2952 (b) and BW4005.2 (c). Genomic coordinates (given in parentheses) and gene orientations (horizontal boxes) are based on the genome sequence of BW2952 [29]. Vertical arrows indicate EcoRV restriction sites, and horizontal arrows show locations of primers used to identify the junction of the duplicated region (Table 2). The triangle shows the location of the 2668-bp deletion in BW4005.2. (d) PFGE of Xbal-digested genomic DNA of BW2952 and BW4005.2. The additional bands in BW4005.2 compared to BW2952 are shown by arrows. (e) RFLP-IS3 and PFGE profiles of six clones (BW4005.C1 to BW4005.C6) derived from the initial glycerol stock of BW4005. The sizes of relevant bands are indicated on the left.

\section{Characterization of a heterozygous duplication by genome re-sequencing}

To address the discrepancies between the different approaches, we decided to re-sequence the genome of BW4005.C6 that harbored the complex rearrangement (Figure 1e). We now detected three mutations compared to BW2952 (Table 1): the same in-frame 3-bp deletion in $a h p C$, the 2668-bp deletion of ogrK-yegR-yegS, and a duplication of $\sim 180 \mathrm{~kb}$ extending from genomic positions $1,993,429$ to $2,172,740$. The duplicated fragment is localized between two IS5 elements (Figure 2), and therefore likely resulted from a recombination event between one and the other of these two IS5 copies during DNA replication. We further validated the presence of the duplication by three additional tests: first, we PCR-amplified and sequenced the new junction sequence resulting from the duplication by using the primers FrontR and FrontF (Table 2, Figure 2). Both the 


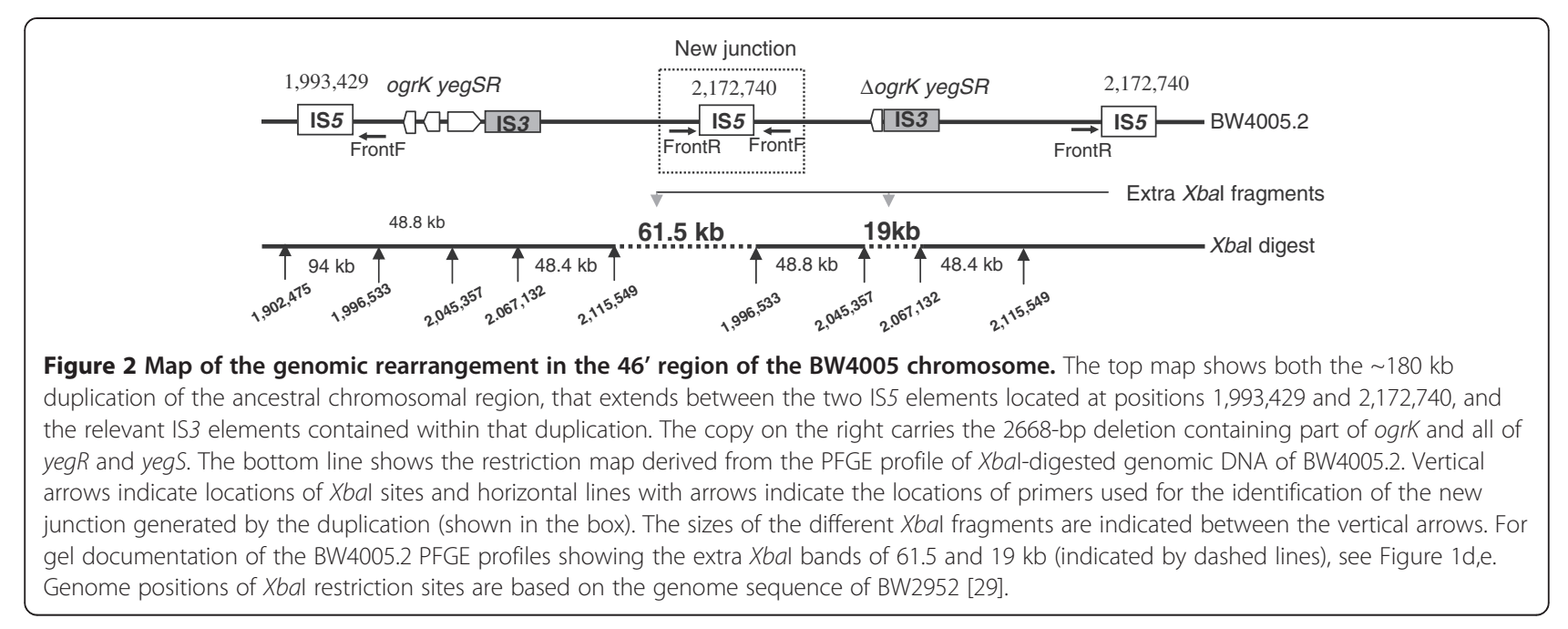

size and sequence of the PCR products confirmed the duplication. Second, an additional IS5-containing EcoRV restriction fragment of $1.5 \mathrm{~kb}$ should be produced at the junction of the duplication (Figure 2) and should therefore be detected by RFLP-IS5. This was indeed shown to be the case (data not shown). Finally, the PFGE profiles with the additional XbaI bands were easily explained on the basis of the duplicated genome sequence (Figure 2).

\section{Phenotypic effects of the rearrangements}

We next investigated the relationships between the complex chromosomal rearrangements and the phenotypes known for BW4005. We measured the growth yield, acetate production, and fitness of the three clones BW4005.C2, BW4005.C3 and BW4005.C6 compared to the ancestor BW2952. These three clones are genotypically different (Figure 1e): the duplication has been resolved into the deleted or ancestral copy in BW4005.C2

Table 2 List of primers used in this study

\begin{tabular}{|c|c|c|c|c|}
\hline $\begin{array}{l}\text { Primer } \\
\text { name }\end{array}$ & Direction & $\begin{array}{l}\text { Genome } \\
\text { position }^{a}\end{array}$ & Sequence $\left(5^{\prime}-3^{\prime}\right)^{b}$ & Used for \\
\hline IS3F1 & Forward & $2,057,888$ & CGCTGTACCGACTCATAAGT & $\begin{array}{l}\text { Detection of ogrk-yegSR } \\
\text { deletion }\end{array}$ \\
\hline IS3R1 & Reverse & $2,061,063$ & GATGCTGAACTCAGCCTGATG & $\begin{array}{l}\text { Detection of ogrK-yegSR } \\
\text { deletion }\end{array}$ \\
\hline IS3R2 & Reverse & $2,059,299$ & CATTCCTTCCTCACGCAAC & $\begin{array}{l}\text { Detection of ogrk-yegSR } \\
\text { deletion }\end{array}$ \\
\hline IS5F1 & Forward & $2,172,688$ & CACCATCAACTGTCTCACCA & Detection of duplication \\
\hline IS5R1 & Reverse & $1,993,645$ & GACCCGCAGATGATGATTAC & Detection of duplication \\
\hline DelF & Forward & $2,057,748$ & CACCGTAACGCTGTTTTGACCG & $\begin{array}{l}\text { Detection of ogrk-yegSR } \\
\text { deletion }\end{array}$ \\
\hline DelR & Reverse & $2,062,009$ & GGATCTTGAGCTCAATTACGCGC & $\begin{array}{l}\text { Detection of ogrk-yegSR } \\
\text { deletion }\end{array}$ \\
\hline IS3EF & Forward & $N A^{c}$ & GCTGCTACGATAATGCCTGCG & Inverse PCR \\
\hline IS3ER & Reverse & $N A^{c}$ & GCGTTCAGCAAGCTTCAGGG & Inverse PCR \\
\hline FrontR & Forward & $1,993,555$ & GTACATTATGCCTGTTCCGAG & Detection of duplication \\
\hline FrontF & Reverse & $2,172,636$ & TCGTATTATTGGCGGTCCC & Detection of duplication \\
\hline ogrKAmpF1 & Forward & $2,057,979$ & GGCTTGTACGCGCATGTGCGGCATGCTGGCATAAGAGTTGGTAGCTCTTGATC & Strain construction \\
\hline yegSAmpF2 & Forward & $2,058,495$ & СTCACTCTCCACATTTGAATGTCAGACGAGCGACAGAGTTGGTAGCTCTTGATC & Strain construction \\
\hline yegRAmpF3 & Forward & $2,059,218$ & CATGGCAGAATTTCCCGCCAGCTTACTGATTCTTAGAGTTGGTAGCTCTTGATC & Strain construction \\
\hline ogrKAmpR1 & Reverse & $2,058,472$ & GGTAAGCGTCAAATATGCGCGTTCTGGCTGTGCCATTCAAATATGTATCCGCT & Strain construction \\
\hline yegSAmpR2 & Reverse & $2,059,189$ & TGATTGGTCTGTAGATAGTGTAGAGCAGAAAACCATTCAAATATGTATCCGCT & Strain construction \\
\hline yegRAmpR3 & Reverse & $2,060,666$ & GACCGTTATTCTCGACAGCGGAAGTACGACAATGCATTCAAATATGTATCCGCT & Strain construction \\
\hline
\end{tabular}

${ }^{\mathrm{a}}$ The positions are given according to the genome sequence of the ancestor strain BW2952 [29]. ${ }^{\mathrm{b}}$ Sequences in bold are homologous to the bla antibiotic resistance gene. 'Not applicable; these primers are complementary to sequences inside the IS3 elements. 
and BW4005.C3, respectively, while BW4005.C6 contains the heterozygous duplication. As shown in Table 3, BW4005.C2 and BW4005.C6 had similar evolved phenotypes (decreased fitness at a 50:50 ratio, reduced yield, and high acetate production under glucose limitation), while BW4005.C3 had phenotypic properties similar to the ancestor. These results suggested that the 2668-bp deletion was the main contributor to the BW4005 evolved phenotypes, and not the $\operatorname{ahpC}$ mutation. Moreover, the presence of the heterozygous duplication seemed to confer similar phenotypes compared to the 2668-bp deletion alone.

We next investigated the respective phenotypic contribution of each of the $y e g R$ and $y e g S$ genes, by first moving the 2668-bp deletion and second inactivating them individually into the ancestral genetic background. We found that both the 2668-bp deletion and yegS-inactivated strains exhibited the evolved phenotypes, while the yegR-inactivated strain had phenotypes indistinguishable from the ancestor (Table 3).

\section{Stability of the duplication}

An important unresolved question is whether the duplication itself conferred a benefit under the chemostat selection conditions. Since evolved phenotypic attributes were not specifically provided by the duplicated state, we tested this indirectly by comparing the population dynamics of the duplication in two environments: non-selective conditions in LB batch cultures and the glucose-limited chemostat selection environment (Figures 1e and 3). The clone BW4005.C6, harboring the heterozygous duplication, was grown either in LB medium for three 24-hour growth cycles or for three days in chemostats in low-frequency competition with MC4100 to mimic the evolution experiment (Materials and methods). In each case, 32 individual clones, sampled at each day interval, were analyzed for the presence of the genomic rearrangements by colony-PCR using the primer pairs IS5F1/IS5R1, IS3F1/IS3R1, and IS3F1/IS3R2. The primer pair IS5F1/IS5R1was specifically designed to detect the new junction produced by the duplication, and IS3F1/IS3R1 and IS3F1/IS3R2 to detect the 2668-bp IS3-mediated deletion (Figure 3a-c). Under the non-selective conditions (Figure 3e), the proportion of clones harboring the heterozygous duplication decreased significantly by the third 24-hour growth cycle ( $t$-test: $P<0.05)$, with the concomitant significant increase of the proportion of clones with single ancestral or deleted copies ( $t$-test: $P<0.05)$. By contrast, the proportion of clones harboring the heterozygous duplication was high and stable over the three days under the original selection condition (Figure 3d). These results showed the genetic instability of the heterozygous duplication under non-selective conditions, in stark contrast to its stability over a short-term period in the glucoselimited conditions prevailing during the evolution experiment. However and despite these stability differences, we were unable to distinguish phenotypically (Table 3 ) the clones harboring the heterozygous duplication and the single deleted copy (even the reconstructed clone with the specific yegS inactivation in the ancestral genetic background had identical evolved phenotypes).

\section{Discussion}

Evolution experiments propagating bacterial populations in glucose- or phosphate-limited chemostats have been extensively developed to understand the dynamics of adaptive processes [19,24-26,28]. Genes involved in transport and consumption of glucose [30-32], encoding global regulators of gene expression [33,34] and DNA repair enzymes $[27,35]$ have been shown to be changed during evolution. However, several evolved clones

Table 3 Phenotypic traits of ancestral, evolved and reconstructed $E$. coli strains

\begin{tabular}{|c|c|c|c|c|c|}
\hline \multirow[t]{2}{*}{ Strains } & \multirow[t]{2}{*}{ Description } & \multirow[t]{2}{*}{ Yield $^{a}$} & \multirow{2}{*}{$\begin{array}{l}\text { Acetate } \\
\text { production }^{b}\end{array}$} & \multicolumn{2}{|c|}{ Fitness $\left(S h^{-1}\right)^{c}$} \\
\hline & & & & $50: 50$ & 1:99 \\
\hline BW2952 & Ancestor & 1.00 & - & 1.00 & 1.00 \\
\hline BW4005.2 & Derivative of evolved isolate BW4005 & $0.89 \pm 0.02$ & + & $-0.04 \pm 0.01$ & $0.06 \pm 0.01$ \\
\hline BW4005.C2 & Derivative of BW4005 (DO) ${ }^{\mathrm{d}}$ & $0.74 \pm 0.08$ & + & $-0.09 \pm 0.01$ & ND \\
\hline BW4005.C3 & Derivative of BW4005 (WT) ${ }^{d}$ & $0.98 \pm 0.03$ & - & $-0.02 \pm 0.01$ & ND \\
\hline BW4005.C6 & Derivative of BW4005 (HD) ${ }^{d}$ & $0.83 \pm 0.05$ & + & $-0.04 \pm 0.01$ & ND \\
\hline BW6029 & BW2952 yegS::bla & $0.85 \pm 0.03$ & + & $-0.02 \pm 0.01$ & $0.09 \pm 0.04$ \\
\hline BW6030 & BW2952 yegR::bla & $1.01 \pm 0.02$ & - & $0.01 \pm 0.01$ & $-0.01 \pm 0.0$ \\
\hline BW6031 & BW2952 AogrK-yegS-yegR::bla & $0.88 \pm 0.02$ & + & $-0.05 \pm 0.01$ & $0.11 \pm 0.04$ \\
\hline
\end{tabular}

${ }^{a}$ Growth yields are shown relative to the ancestral strain BW2952 and were obtained from 24-hour glucose-limited chemostats by measuring the optical density of the cultures at $600 \mathrm{~nm}$. Values are from three independent measurements.

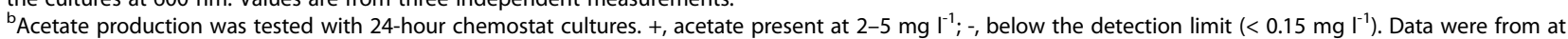
least three independent biological repeats with less than $10 \%$ standard errors.

${ }^{c}$ Competing strains were mixed at 50:50 or 1:99 ratios after individual acclimation in the chemostat evolution conditions. Changes in the proportions of the competitors after 24 hours were used to calculate fitness values. Data presented are mean \pm SEM from at least two independent experiments. ND, not determined.

${ }^{\mathrm{d}}$ Symbols are identical to the legend of Figure 3. 
a

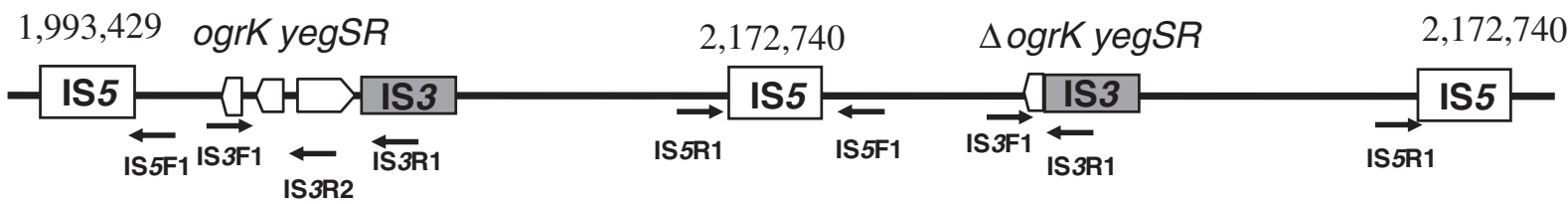

b

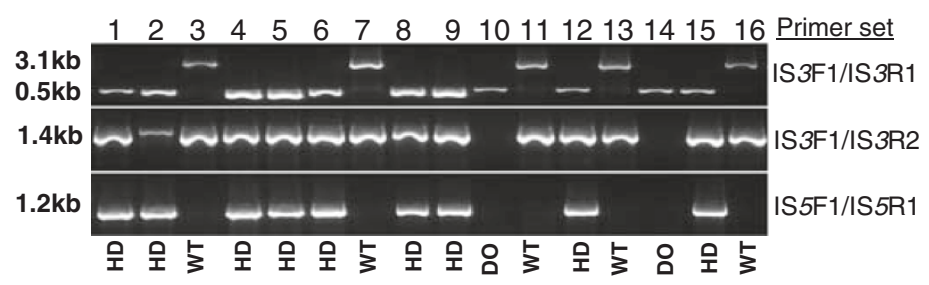

C

\begin{tabular}{|lccc|}
\hline \multirow{2}{*}{ Genotype } & \multicolumn{2}{c}{ Primers } & PCR product(s) \\
\cline { 2 - 3 } & Forward & Reverse & $(\mathrm{kb})$ \\
\hline \multirow{3}{*}{ Heterozygous duplication } & IS5F1 & IS5R1 & 1.2 \\
& IS3F1 & IS3R1 & $0.5 \& 3.1^{*}$ \\
& IS3F1 & IS3R2 & 1.4 \\
\hline \multirow{3}{*}{ Resolved deleted copy } & IS5F1 & IS5R1 & No product \\
& IS3F1 & IS3R1 & 0.5 \\
& IS3F1 & IS3R2 & No product \\
\hline \multirow{3}{*}{ Resolved ancestral copy } & IS5F1 & IS5R1 & No product \\
& IS3F1 & IS3R1 & 3.1 \\
& IS3F1 & IS3R2 & 1.4 \\
\hline
\end{tabular}

d
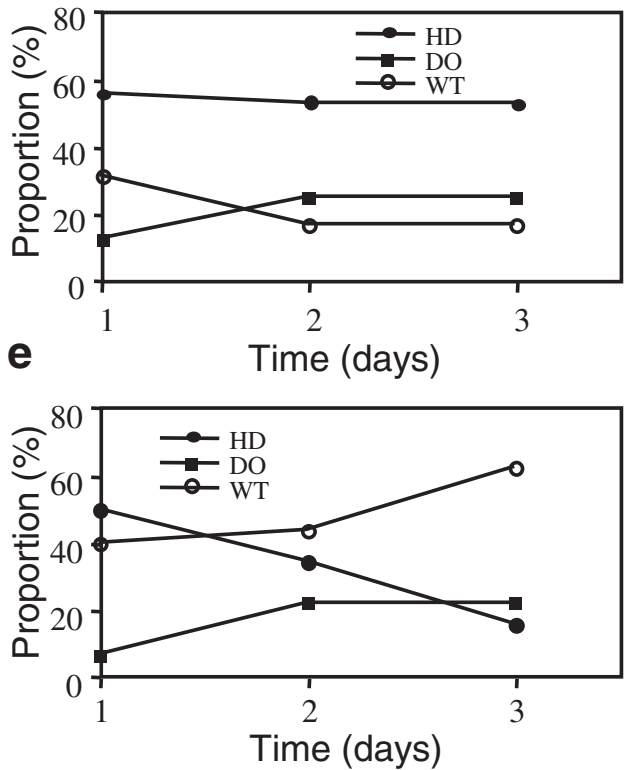

Figure 3 Stability of the duplication and phenotypes of the different derived genotypes. (a) Chromosomal map of the duplication in BW4005 showing primer pairs used to follow the fate of the duplication. The same symbols as in Figure 2 are used. (b) Example of PCR experiments to score the state of the genomic rearrangement, by using the three primer pairs IS5F1/IS5R1, IS3F1/IS3R1 and IS3F1/IS3R2. WT and DO stand for the presence of the single ancestral or 2668-bp deleted copy, respectively, and HD for the presence of the heterozygous duplication. The expected results for each of the three genotypes are shown in (c). ${ }^{*}$ Owing to the higher efficiency of production of the smaller $0.5 \mathrm{~kb}$ PCR product with IS3F1/IS3R1, we had difficulties in detecting simultaneously the larger $3.1 \mathrm{~kb}$ PCR product in clones harboring the heterozygous duplication. We therefore also performed PCR reactions using the additional primer pair IS3F1/IS3R2 to improve the accuracy of scoring the heterozygous duplication state. (d,e) Stability of the heterozygous duplication under the selective conditions of the chemostat (d) and non-selective conditions of LB batch cultures (e). In (d), BW4005.C6 harboring the heterozygous duplication was mixed 1:99 with the MC4100 reference strain after individual acclimation in separate chemostats. Samples were taken every 24 hours for three days and appropriate dilutions were plated onto LB plates with or without $30 \mathrm{~g} \mathrm{ml}^{-1}$ kanamycin. At each time point, we sampled 32 well-separated colonies from Kan-plates and scored them for the IS5-mediated duplication and IS3-mediated deletion. In (e), an overnight culture of BW4005.C6 was diluted 100 -fold into LB liquid medium and grown for 24 hours. This growth cycle was repeated for two additional days and the proportion of the different genotypes was followed as in (b). The data presented are the average of two independent cultures in each case.

followed alternative evolutionary pathways not uncovered by whole-genome sequencing. Here, we identified one such path, consisting of complex chromosomal rearrangements that included a large 180-kb duplication of the $46^{\prime}$ chromosomal region followed by the divergence of the two tandem duplicated copies. One copy experienced a 2668-bp deletion removing part of the ogrK gene and both $y e g R$ and $y e g S$. We showed that the duplication was highly unstable under non-selective conditions but not in the conditions prevailing during evolution. However, the duplication per se did not provide any of the phenotypic traits associated with the adaptation of these evolved clones in the chemostats. In striking contrast, inactivating yegS in the ancestral background was sufficient to confer all evolved phenotypes.

The benefit conferred by the yegS deletion, together with the dominance of the deleted allele over its ancestral counterpart, are difficult to infer owing to the lack of information about YegS. Indeed, YegS is annotated as a putative diacylglycerol kinase potentially involved in the response to acid stress [36]. A yegS knockout results in the loss of mannose and acetate consumption [37]. Diacylglycerol is a lipid second messenger in many organisms [38], and the yegS deletion may result in alterations of regulatory and/or metabolic networks owing to changes in lipid signaling pathways in $E$. coli. 
Previous studies, with Salmonella typhimurium populations propagated in arabinose- or melibiose-limited chemostats [22], detected 96 and 25\%, respectively, of cells harboring araE or $\mathrm{melB}$ duplications that emerged within only 50-100 hours of incubation. The amplified genes are responsible for arabinose or melibiose uptake and are therefore under strong selection for elevated expression levels under these conditions. In Salmonella, duplications and rearrangements also occur upon prolonged storage of strains and in clinical settings $[39,40]$. In contrast, in the glucose-limited chemostat populations of $E$. coli, the major early adaptive changes involve a series of regulatory mutations increasing the levels of glucose transport components [19,24-26,28,41]. Earlier studies of lactose-limited chemostats also showed that the first selective sweeps were by lac-constitutive mutants, only later followed by DNA amplifications [4]. Therefore, one may speculate whether inherent differences between Salmonella and E. coli may exist for duplication rates and/or costs. Interestingly, the BW4005 lineage carrying the duplication emerged later as a minority clone, with an adaptive pathway very different from the other clones in the chemostat population [19], since its fitness was shown to be frequency dependent.

While many duplication (and amplification) events have been detected in classical genetic studies where strong selection was applied, it is not the case during laboratory evolution experiments, with one notable exception $[16,17]$. We showed here that this may be merely linked to the high instability of the duplication events under non-selective conditions. Hence, a single overnight growth cycle was sufficient to generate at high frequency offspring that lost the duplication (Figure 1e). Most analyses of evolved clones sampled from evolution experiments involve culture cycles under non-selective conditions for further analyses. We show here that such experimental practices may result in the loss of duplication events and that sampling single clones may lead to an underestimation of these chromosomal rearrangements. Therefore, storing and analyzing evolved isolates must be carefully performed. Instabilities in bacterial stocks are well-known $[39,42,43]$ and our data emphasize the importance of minimizing sub-culturing steps. It was only good fortune that at least one clone, randomly sampled from the original chemostat population, retained the duplication.

A comprehensive view of the stability of genome duplications is yet missing at the evolutionary, ecological and molecular levels. The RecA protein is involved in homologous recombination and is important in resolving duplications [44]. On the other hand, some bacterial duplications may be stable, including those involving recombination at rRNA ( $r r n)$ operons $[39,45]$. Here, we observed that the heterozygous duplication was much more stable under the glucose-limited chemostat conditions than under non-selective conditions (Figure 3). Therefore, it might confer a direct selective advantage in the evolution regime. However, all evolved phenotypes were related to either the 2668-bp deletion alone or the single inactivation of yegS. Hence, the duplication by itself does probably not confer a direct benefit to the evolved cells, in contrast to duplications that result in a gene-dosage advantage [1-3].

Several reasons may explain the stability differences in selective and non-selective conditions. First, the resolution components may be differentially regulated in various environments. Second, the fitness cost of the duplication may be larger during rapid growth resulting in higher instability. Moreover, we showed that the heterozygous duplication had no fitness disadvantage over the single deletion copy, suggesting little fitness cost in chemostats. Third, the duplication may represent a precursor enhancing the likelihood of the substitution of a beneficial mutation through an increase in the mutational target and in local mutation rates. As such, the contribution of the duplication in the evolution of BW4005 may involve a second-order selection process [46] that has been shown to occur during experimental evolution with E. coli [47]. Finally, our results are not consistent with the model for divergence after duplication [2], in which selection continuously favors both the maintenance of the duplicated copies and the divergence of one copy from the parental one.

A fascinating property of the evolved duplication is its ability to resolve into either the ancestral or deleted copy that may provide robustness by buffering against deleterious mutations. On the other hand, the capacity to recover the ancestral chromosome when selection is removed endows the evolved strain with increased evolvability in alternative environments. Finally, the demonstration of ancestral and evolved copies inside a duplication provides a newly documented example of an evolutionary intermediate, with different selection pressures acting on the two copies providing the basis of evolutionary divergence.

\section{Conclusions}

We identified for the first time during laboratory evolution experiments the presence of a heterozygous duplication, one copy presenting a small deletion that was shown to be dominant and beneficial under the selective conditions. Studies of the stability of the duplicated genotype under different environmental conditions revealed that it was unlikely to have been submitted to direct selection during evolution but rather to indirect selection. This duplication therefore balances the robustness and evolvability traits of the bacterial populations evolving in the glucose-limited chemostats. 


\section{Methods}

All bacterial strains used in this study are listed in Table 4. The E. coli K-12 strain BW2952 [29], a kanamycinresistant derivative of $\mathrm{MC4100,}$ was used as the ancestor to initiate glucose-limited chemostats that were fed with $0.02 \% \mathrm{wt} / \mathrm{vol}$ glucose in minimal medium A [48] with a $0.1 \mathrm{~h}^{-1}$ dilution rate, as previously described [49]. This study focused on the BW4005 evolved strain, one of 41 randomly sampled clones from the 26-day sample (representing $\sim 90$ generations of evolution) of one such chemostat [24]. These 41 evolved clones were preserved as glycerol suspensions at $-80^{\circ} \mathrm{C}$ directly after sampling. During this study, eight clones, called respectively BW4005.1, BW4005.2 and BW4005.C1 to BW4005.C6, were directly derived from the initial glycerol stock of BW4005.

Competitions in chemostats were performed between the kanamycin-resistant, tetracycline-sensitive evolved clone BW4005.C6 derived from BW2952 and the kanamycin-susceptible tetracycline-resistant BW3454

Table 4 List of strains used in this study

\begin{tabular}{|c|c|c|}
\hline Strain & Relevant genotype & Source \\
\hline MC4100 & $\begin{array}{l}\mathrm{F}^{-} \text {araD139 } \Delta \text { (argF-lac) U169 rspL150 deoCl relA1 thiA } \\
\text { ptsF25 flb5301 rbsR }\end{array}$ & {$[29]$} \\
\hline BW2952 & MC4100 malG::^placMu55f(malG:.:acZ) & {$[29]$} \\
\hline BW3454 & MC4100 metC162:::Tn10 & {$[25]$} \\
\hline BW4005 & $\begin{array}{l}\text { Chemostat evolved clone sampled after } 90 \\
\text { generations }\end{array}$ & {$[19,24]$} \\
\hline $\begin{array}{l}\text { BW4005. } \\
\text { C1 }\end{array}$ & Colony 1 derived from BW4005 & $\begin{array}{l}\text { This } \\
\text { study }\end{array}$ \\
\hline $\begin{array}{l}\text { BW4005. } \\
\text { C2 }\end{array}$ & Colony 2 derived from BW4005 & $\begin{array}{l}\text { This } \\
\text { study }\end{array}$ \\
\hline $\begin{array}{l}\text { BW4005. } \\
\text { C3 }\end{array}$ & Colony 3 derived from BW4005 & $\begin{array}{l}\text { This } \\
\text { study }\end{array}$ \\
\hline $\begin{array}{l}\text { BW4005. } \\
\text { C4 }\end{array}$ & Colony 4 derived from BW4005 & $\begin{array}{l}\text { This } \\
\text { study }\end{array}$ \\
\hline $\begin{array}{l}\text { BW4005. } \\
\text { C5 }\end{array}$ & Colony 5 derived from BW4005 & $\begin{array}{l}\text { This } \\
\text { study }\end{array}$ \\
\hline $\begin{array}{l}\text { BW4005. } \\
\text { C6 }\end{array}$ & Colony 6 derived from BW4005 & $\begin{array}{l}\text { This } \\
\text { study }\end{array}$ \\
\hline DY330 & 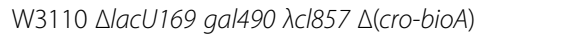 & {$[50]$} \\
\hline BW6026 & DY330 yegS::bla & $\begin{array}{l}\text { This } \\
\text { study }\end{array}$ \\
\hline BW6027 & DY330 yegR::bla & $\begin{array}{l}\text { This } \\
\text { study }\end{array}$ \\
\hline BW6028 & DY330 ogrk-yegS-yegR::bla & $\begin{array}{l}\text { This } \\
\text { study }\end{array}$ \\
\hline BW6029 & BW2952 yegs::bla & $\begin{array}{l}\text { This } \\
\text { study }\end{array}$ \\
\hline BW6030 & BW2952 yegR::bla & $\begin{array}{l}\text { This } \\
\text { study }\end{array}$ \\
\hline BW6031 & BW2952 $\Delta$ ogrk-yegS-yegR::bla & $\begin{array}{l}\text { This } \\
\text { study }\end{array}$ \\
\hline
\end{tabular}

strain, which was used as the common reference ancestral competitor.

Outside chemostats, strains were grown in LB liquid or solid medium. Allelic exchange was performed using $\mathrm{LB}+$ sucrose plates without $\mathrm{NaCl}$ and supplemented with $50 \%$ sucrose. When necessary, antibiotics were added: chloramphenicol or kanamycin each at $30 \mu \mathrm{g} \mathrm{ml}^{-1}$, and ampicillin at $100 \mu \mathrm{g} \mathrm{ml}^{-1}$.

\section{Strain construction}

The 2668-bp deletion from the BW4005 evolved clone was moved into the ancestral genome by allelic exchange using the suicide plasmid pKO3 [51]. Briefly, a 1100-bp PCR fragment with about 550 bp of adjacent sequences on each side of the deletion was obtained using BW4005 genomic DNA and the primers DelR and DelF (Table 2), and then cloned into pKO3. The obtained plasmid was introduced by electro-transformation into BW2952 and chloramphenicol-resistant cells, formed after chromosomal integration of the plasmid, were selected. Resistant clones were streaked onto LB+sucrose plates to select for cells that lost the plasmid. Indeed, pKO3 carries the $s a c B$ gene that makes cells carrying that plasmid susceptible to killing by sucrose. These cells were then screened by PCR and scored for the presence of the deletion allele by the size of the PCR products. We constructed yegS::bla and yegR::bla derivatives of BW2952 using homologous flanking sequences as described [50]. The primer sets used for strain constructions are listed in Table 2 .

\section{Phenotypic assays}

Analysis of extracellular acetate in culture media from individual chemostats inoculated with different bacterial strains was performed after filtration through a Milex ${ }^{\circ}$ filter unit (Millpore, Ireland, $0.22 \mu \mathrm{m}$ ) by using an assay kit for acetic acid with the UV method (Boehringer Mannheim/R-Biopharm, Germany), according to the manufacturer's guidelines. The growth yields were inferred by measuring the optical density at $600 \mathrm{~nm}$ of 24-hour chemostat cultures growing in the exact same conditions prevailing during the evolution experiment.

\section{Pairwise competition experiments and fitness estimation}

Each bacterial strain was competed against a metC::Tn10 derivative, called BW3454 (Table 4), of the BW2952 ancestral strain [29], under the same conditions used during the chemostat evolution experiment, except that the medium was supplemented with $4 \mu \mathrm{g} \mathrm{ml} \mathrm{m}^{-1}$ methionine. Before mixing the two competitors, each strain was independently acclimated by overnight growth in the glucoselimited chemostats to ensure identical physiological state. The fitness of the competing strains was calculated as the selection coefficient $S$, as described [19]. 


\section{Genome re-sequencing}

A first single colony of BW4005, called BW4005.1, was isolated after streaking the original glycerol suspension on $\mathrm{LB}$ plate and incubating overnight at $37^{\circ} \mathrm{C}$. The BW4005.1 clone was then inoculated into $10 \mathrm{ml} \mathrm{LB}$ and grown overnight at $37^{\circ} \mathrm{C}$. This culture was used for genomic DNA extraction as described [29]. Whole genome re-sequencing of BW4005.1 was performed by 454 and single-end Solexa technologies [33]. Briefly, a total of 216,058 single-end reads with an average length of 404 bps, giving a 19.08X coverage of the genome, were generated using the GS FLX system (Roche, Welwyn Garden City, Hertfordshire, UK) and assembled to 78 contigs with 454 Newbler assembler (http://www.454. com/products/analysis-software/). A total of $8,862,831$ single-end reads with an average length of 33 bps were generated using the Solexa Genome Analyzer (Illumina, Little Chesterford, Essex), giving a $63.9 \times$ coverage. All Solexa reads were mapped to the contigs generated from Newbler using MAQ [52]. The order of the contigs was determined based on BLASTn alignments with the genome sequence of BW2952 as the reference (Genbank accession number NC_012759). By analyzing these data, we found a few sequencing errors in the original BW2952 genome sequence (Additional file 1: Table S1). All 78 sequence gaps in BW4005 and 151 suspected SNP sites were filled or checked by sequencing PCR products with ABI 3730XL capillary sequencer (Applied Biosystems, Foster City, California, USA).

In our second genome re-sequencing attempt, a second colony derived independently from the same initial glycerol stock of BW4005, and called BW4005.C6 (see below, Table 4), was grown as described above. (BW4005.C6 was confirmed by our molecular analyses to harbor the heterozygous duplication). Its genomic DNA was extracted, checked and re-sequenced on the Illumina Genome Analyzer platform using single-end 36-bp reads (GATCBiotech, Germany). Candidate mutations were identified in comparison to the ancestral BW2952 genome [29] using a recently developed computational pipeline [17]. Genome sequencing data have been deposited in the ENA Sequence Read Archive (ERP002251).

\section{RFLP-IS and characterization of IS adjacent sequences by inverse PCR}

Restriction fragment length polymorphism (RFLP) using internal fragments of IS3 and IS5 as probes (RFLP-IS3 and RFLP-IS5, respectively) was performed with genomic DNA of BW2952, BW4005 and derived clones as described previously [27]. Genomic sequences adjacent to relevant IS3 elements were identified by inverse PCR experiments [27], using primers IS3ER and IS3EF (Table 2) that are complementary to the extremities of IS3 and oriented toward its flanking regions. Briefly, after EcoRV digestion of genomic DNA from clone BW4005.2 and fractionation by agarose gel electrophoresis, the relevant DNA fragments were extracted from the gel, self-ligated and submitted to PCR using the primers IS3ER and IS3EF. The obtained PCR products were then sequenced to identify the IS3 genomic neighborhood.

\section{Detection of the junction fragment of the duplication and analysis of the duplication stability}

The new junction fragment generated by the duplication event was detected by PCR experiments using the two primer pairs FrontR/FrontF and IS5F1/IS5R1 (Table 2). The stability of the duplication was assessed by subculturing the evolved clone BW4005 in two different environments: non-selective LB medium and the chemostat evolution conditions. For non-selective conditions, the initial glycerol stock of BW4005 was plated onto LB solid media and incubated overnight at $37^{\circ} \mathrm{C}$. Six colonies were sampled and grown for 12 hours in LB liquid medium at $37^{\circ} \mathrm{C}$, the resulting clones being called BW4005.C1 to BW4005.C6. The BW4005.C6 clone, that was shown to retain the duplication after culture, was further inoculated by a 1:100 dilution into two independent McCartney bottles, each containing $5 \mathrm{ml} \mathrm{LB}$, and incubated for 24 hours at $37^{\circ} \mathrm{C}$ with shaking at 200 $\mathrm{rpm}$. These dilution/growth cycles were repeated for a total of three times. At each of the three steps, $100 \mu \mathrm{l}$ of the appropriately diluted 24-hour cultures were plated onto LB solid medium and 32 well-separated colonies, 16 deriving from each of the two independent McCartney bottles, were sampled for DNA extraction and subsequent PCR analysis to score for the presence or absence of the duplication. For selective chemostat conditions, glucose-limited chemostats of BW4005.C6 and MC4100 were mixed after 16 hours of acclimation. Owing to the negative frequency-dependent fitness and low frequency of BW4005 in the initial chemostat evolution experiment [19], we mixed BW4005.C6 with MC4100 at a ratio of $1: 99$ and this co-culture was maintained for three days. Once every day, we sampled 32 colonies derived from BW4005.C6 after plating appropriate dilutions on LB plates and scoring for colonies resistant to kanamycin.

\section{Pulsed-field gel electrophoresis (PFGE)}

PFGE was performed using a CHEF Mapper system (Bio-Rad Laboratories, Hercules, CA, USA) according to the procedure described by the US CDC PulseNet program (http://www.cdc.gov/pulsenet/pathogens/pfge. html). XbaI-digested genomic DNAs of BW2952, BW4005 and derived clones were separated at $6.0 \mathrm{~V} / \mathrm{cm}$ for 19 hours at $14^{\circ} \mathrm{C}$, with increasing pulse times of $6-36 \mathrm{~s}$ and an angle of $120^{\circ}$. 


\section{Additional file}

Additional file 1: Table S1. lists the sequencing errors that were detected in the genome of the ancestor BW2952 that was previously sequenced [29].

\section{Abbreviations}

PFGE: Pulsed-field gel electrophoresis; RFLP: Restriction fragment length polymorphism; RFLP-IS3: Restriction fragment length polymorphism with IS3 as probe; RFLP-IS5: Restriction fragment length polymorphism with IS5 as probe; SNP: Single-nucleotide polymorphism.

\section{Competing interests}

The authors declare that they have no competing interests.

\section{Authors' contributions}

RM carried out the physiological and fitness assays on all clones and constructed knockout strains. JG performed the RFLP-IS experiments. JG and JP constructed mutant strains. MS performed PFGE experiments. LW and LF were responsible for the genome sequencing and comparative genomics studies of BW4005.1. OT analyzed the genome sequence of BW4005.C6. DS and TF conceived the study, its design and coordination, and wrote the manuscript. All authors read and approved the final manuscript.

\section{Acknowledgements}

We thank the Australian Research Council for grant support (to TF). This work was also supported by the National Basic Research Program of China (973 Program) [2009CB522603] and the National Natural Science Foundation of China (NSFC) Key Program [31030002] (to LW and LF); the Agence Nationale de la Recherche (ANR) Program "Blanc" (ANR-08-BLAN-0283-01, to DS), Centre National de la Recherche Scientifique, and Université Joseph Fourier (to DS); and the Agence Nationale de la Recherche (ANR) Program "Génomique" (ANR-08-GENM-023, to DS and OT). JP thanks the French Ministry of Research and the Université Joseph Fourier for a PhD fellowship.

\section{Author details}

${ }^{1}$ School of Molecular Bioscience, University of Sydney, Sydney, NSW 2006, Australia. ' 2 aboratoire Adaptation et Pathogénie des Micro-organismes, Université Joseph Fourier Grenoble, BP 170, F-38042, Grenoble cedex 9 , France. ${ }^{3}$ CNRS UMR5163, F-38042, Grenoble cedex 9, France. ${ }^{4}$ TEDA School of Biological Sciences and Biotechnology, Nankai University, 23 Hongda Street, Tianjin 300457, P.R. China. ${ }^{5}$ Key Laboratory of Molecular Microbiology and Technology, Ministry of Education, 23 Hongda Street, Tianjin 300457, P. R. China. ${ }^{6}$ INSERM, UMR-S 722, F-75018, Paris, France. ${ }^{7}$ Université Paris Diderot, Sorbonne Paris Cité, UMR-S 722 INSERM, F-75018, Paris, France.

Received: 22 October 2012 Accepted: 8 March 2013 Published: 3 July 2013

\section{References}

1. Kugelberg E, Kofoid E, Reams AB, Andersson DI, Roth JR: Multiple pathways of selected gene amplification during adaptive mutation. Proc Natl Acad Sci USA 2006, 103:17319-17324.

2. Sun $\mathrm{S}$, Berg OG, Roth JR, Andersson DI: Contribution of gene amplification to evolution of increased antibiotic resistance in Salmonella typhimurium. Genetics 2009, 182:1183-1195.

3. Andersson DI, Hughes D: Gene amplification and adaptive evolution in bacteria. Annu Rev Genet 2009, 43:167-195.

4. Novick A, Horiuchi T: Hyper-production of beta-galactosidase by Escherichia coli bacteria. Cold Spring Harbor Symp Quant Biol 1961, 26:239-245.

5. Brown CJ, Todd KM, Rosenzweig RF: Multiple duplications of yeast hexose transport genes in response to selection in a glucose-limited environment. Mol Biol Evol 1998, 15:931-942.

6. Dunham MJ, Badrane H, Ferea T, Adams J, Brown PO, Rosenzweig F, Botstein D: Characteristic genome rearrangements in experimental evolution of Saccharomyces cerevisiae. Proc Natl Acad Sci USA 2002, 99:16144-16149.
7. Wagner A: The role of population size, pleiotropy and fitness effects of mutations in the evolution of overlapping gene functions. Genetics 2000 154:1389-1401.

8. Zhang J: Evolution by gene duplication: an update. Trends Ecol Evol 2003 18:292-298.

9. Blount ZD, Barrick JE, Davidson CJ, Lenski RE: Genomic analysis of a key innovation in an experimental Escherichia coli population. Nature 2012, 489:513-518.

10. Gevers D, Vandepoele K, Simillion C, Van de Peer Y: Gene duplication and biased functional retention of paralogs in bacterial genomes. Trends Microbiol 2004, 12:148-154.

11. Taylor JS, Raes J: Duplication and divergence: The evolution of new genes and old ideas. Annu Rev Genet 2004, 38:615-643.

12. Ohno S: Evolution by Gene Duplication. New York: Springer-Verlag; 1970.

13. Afriat $L$, Roodveldt C, Manco G, Tawfik DS: The latent promiscuity of newly identified microbial lactonases is linked to a recently diverged phosphotriesterase. Biochemistry 2006, 45:13677-13686.

14. Force A, Lynch M, Pickett FB, Amores A, Yan Y-I, Postlethwait J: Preservation of duplicate genes by complementary, degenerative mutations. Genetics 1999, 151:1531-1545

15. Hindre T, Knibbe C, Beslon G, Schneider D: New insights into bacterial adaptation through in vivo and in silico experimental evolution. Nat Rev Microbiol 2012, 10:352-365.

16. Riehle MM, Bennett AF, Long AD: Genetic analysis of the adaptation to temperature stress: a role for gene duplications. Am Zool 2000, 40:1188-1189.

17. Tenaillon $O$, Rodriguez-Verdugo A, Gaut RL, McDonald P, Bennett AF, Long $A D$, Gaut $B S$ : The molecular diversity of adaptive convergence. Science 2012, 335:457-461.

18. Barrick JE, Yu DS, Yoon SH, Jeong H, Oh TK, Schneider D, Lenski RE, Kim JF: Genome evolution and adaptation in a long-term experiment with Escherichia coli. Nature 2009, 461:1243-1247.

19. Maharjan R, Ferenci T, Reeves $P$, Li Y, Liu B, Wang L: The multiplicity of divergence mechanisms in a single evolving population. Genome Biol 2012, 13:R41.

20. Herring CD, Raghunathan A, Honisch C, Patel T, Applebee MK, Joyce AR, Albert TJ, Blattner FR, van den Boom D, Cantor CR, Palsson BO: Comparative genome sequencing of Escherichia coli allows observation of bacterial evolution on a laboratory timescale. Nat Genet 2006, 38:1406-1412

21. Velicer GJ, Raddatz G, Keller H, Deiss S, Lanz C, Dinkelacker I, Schuster SC: Comprehensive mutation identification in an evolved bacterial cooperator and its cheating ancestor. Proc Natl Acad Sci USA 2006, 103:8107-8112.

22. Sonti RV, Roth JR: Role of gene duplications in the adaptation of Salmonella typhimurium to growth on limiting carbon sources. Genetics 1989, 123:19-28.

23. Ferenci T: Bacterial physiology, regulation and mutational adaptation in a chemostat environment. Adv Microb Physiol 2008, 53:169-229.

24. Maharjan R, Seeto S, Notley-McRobb L, Ferenci T: Clonal adaptive radiation in a constant environment. Science 2006, 313:514-517.

25. Wang L, Spira B, Zhou ZM, Feng L, Maharjan RP, Li XM, Li FF, McKenzie C, Reeves PR, Ferenci T: Divergence involving global regulatory gene mutations in an Escherichia coli population evolving under phosphate limitation. Genome Biol Evol 2010, 2:478-487.

26. Kinnersley MA, Holben WE, Rosenzweig F: E Unibus Plurum: Genomic analysis of an experimentally evolved polymorphism in Escherichia coli. PLoS Genet 2009, 5(11):e1000713.

27. Gaffe J, McKenzie C, Maharjan RP, Coursange E, Ferenci T, Schneider D: Insertion sequence-driven evolution of Escherichia coli in chemostats. J Mol Evol 2011, 72:398-412.

28. Maharjan R, Seeto S, Ferenci T: Divergence and redundancy of transport and metabolic rate-yield strategies in a single Escherichia coli population. J Bacteriol 2007, 189:2350-2358.

29. Ferenci T, Zhou ZM, Betteridge T, Ren Y, Liu Y, Feng L, Reeves PR, Wang L: Genomic sequencing reveals regulatory mutations and recombinational events in the widely used MC4100 lineage of Escherichia coli K-12. J Bacteriol 2009, 191:4025-4029.

30. Maharjan R, McKenzie C, Yeung A, Ferenci T: The basis of antagonistic pleiotropy in hfq mutations that have opposite effects on fitness at slow and fast growth rates. Heredity 2013, 110:10-18. 
31. Notley-McRobb L, Ferenci T: Adaptive $m g$-regulatory mutations and genetic diversity evolving in glucose-limited Escherichia coli populations. Environ Microbiol 2013, 111:10-18.

32. Notley-McRobb L, Ferenci T: The generation of multiple coexisting malregulatory mutations through polygenic evolution in glucose-limited populations of Escherichia coli. Environ Microbiol 1999, 1:45-52.

33. Maharjan R, Zhou ZM, Ren Y, Li Y, Gaffe J, Schneider D, McKenzie C, Reeves $P R$, Ferenci T, Wang L: Genomic identification of a novel mutation in $h f a$ that provides multiple benefits in evolving glucose-limited populations of Escherichia coli. J Bacteriol 2010, 192:4517-4521.

34. Notley-McRobb L, King T, Ferenci T: rpoS mutations and loss of general stress resistance in Escherichia coli populations as a consequence of conflict between competing stress responses. J Bacteriol 2002, 184:806-811.

35. Notley-McRobb L, Seeto S, Ferenci T: Enrichment and elimination of mutY mutators in Escherichia coli populations. Genetics 2002, 162:1055-1062.

36. Bakali HMA, Herman MD, Johnson KA, Kelly AA, Wieslander $\AA$, Hallberg BM, Nordlund P: Crystal structure of YegS, a homologue to the mammalian diacylglycerol kinases, reveals a novel regulatory metal binding site. J Biol Chem 2007, 282:19644-19652.

37. Ito M, Baba T, Mori H: Functional analysis of 1440 Escherichia coli genes using the combination of knock-out library and phenotype microarrays. Metab Eng 2005, 7:318-327.

38. Almena M, Mérida I: Shaping up the membrane: diacylglycerol coordinates spatial orientation of signaling. Trends Biochem Sci 2011, 36:593-603.

39. Porwollik S, Wong RM-Y, Helm RA, Edwards KK, Calcutt M, Eisenstark A, McClelland M: DNA amplification and rearrangements in archival Salmonella enterica Serovar Typhimurium LT2 cultures. J Bacteriol 2004, 186:1678-1682.

40. Matthews TD, Rabsch W, Maloy S: Chromosomal rearrangements in Salmonella enterica Serovar Typhi strains isolated from asymptomatic human carriers. mBio 2011, 2:e00060-11.

41. Notley-McRobb L, Ferenci T: Experimental analysis of molecular events during mutational periodic selections in bacterial evolution. Genetics 2000, 156:1493-1501.

42. Faure D, Frederick R, Wloch D, Portier P, Blot M, Adams J: Genomic changes arising in long-term stab cultures of Escherichia coli. J Bacteriol 2004, 186:6437-6442. e00060-11.

43. Spira B, de Almeida TR, Maharjan R, Ferenci T: The uncertain consequences of transferring bacterial strains between laboratories - rpoS instability as an example. BMC Microbiol 2011, 11:248.

44. Reams AB, Kofoid E, Savageau M, Roth JR: Duplication frequency in a population of Salmonella enterica rapidly approaches steady state with or without recombination. Genetics 2010, 184:1077-1094.

45. Liu SL, Sanderson KE: The chromosome of Salmonella paratyphi A is inverted by recombination between $\mathrm{rrnH}$ and $\mathrm{rrnG}$. J Bacteriol 1995, 177:6585-6592.

46. Tenaillon O, Taddei F, Radman M, Matic I: Second-order selection in bacterial evolution: selection acting on mutation and recombination rates in the course of adaptation. Res Microbiol 2001, 152:11-16.

47. Woods RJ, Barrick JE, Cooper TF, Shrestha U, Kauth MR, Lenski RE: Secondorder selection for evolvability in a large Escherichia coli population. Science 2011, 331:1433-1436.

48. Miller J: Experiments in Molecular Genetics. Cold Spring Harbor, N.Y. Cold Spring Harbor Laboratory; 1972.

49. Notley-McRobb L, Seeto S, Ferenci T: The influence of cellular physiology on the initiation of mutational pathways in Escherichia coli populations. Proc R Soc Lond Ser B-Biol Sci 2003, 270:843-848.

50. Yu DG, Ellis HM, Lee EC, Jenkins NA, Copeland NG, Court DL: An efficient recombination system for chromosome engineering in Escherichia coli. Proc Natl Acad Sci USA 2000, 97:5978-5983.

51. Link AJ, Phillips D, Church GM: Methods for generating precise deletions and insertions in the genome of wild-type Escherichia coli - application to open reading frame characterization. J Bacterio/ 1997, 179:6228-6237.

52. Li H, Ruan J, Durbin R: Mapping short DNA sequencing reads and calling variants using mapping quality scores. Genome Res 2008, 18:1851-1858.

doi:10.1186/1471-2164-14-441

Cite this article as: Maharjan et al:: A case of adaptation through a mutation in a tandem duplication during experimental evolution in Escherichia coli. BMC Genomics 2013 14:441.

\section{Submit your next manuscript to BioMed Central and take full advantage of:}

- Convenient online submission

- Thorough peer review

- No space constraints or color figure charges

- Immediate publication on acceptance

- Inclusion in PubMed, CAS, Scopus and Google Scholar

- Research which is freely available for redistribution 